\title{
Diagnosis of Keratoconus with Corneal Features Obtained through LBP, LDP, LOOP and CSO
}

\section{P Subramanian* and GP Ramesh}

\author{
Department of Electronics and Communication Engineering, St. Peter's Institute of Higher Education \\ and Research, Chennai, India
}

(Corresponding author's e-mail: subramanian602@gmail.com)

Received: 13 March 2021, Revised: 19 May 2021, Accepted: 23 May 2021

\begin{abstract}
Keratoconus, by its name, is the condition of the eye wherein the cornea assumes a conical shape due to the thinning and protrusion of the cornea. Keratoconus, though bilateral, can be asymmetric in that it can progress differently in the eyes of the patient. Keratoconus can start from early adulthood and progress till the age of 40. Early detection of keratoconus is vital in preventing vision loss or costly repairs. The diagnostic tools available range from keratoscope to videokeratoscope but involves human efforts and thereby human errors. Automatic detection of keratoconus is required for large screening camps. With the advances in artificial intelligence techniques for medical diagnosis, new algorithms and techniques have been developed for the early and rapid screening of keratoconus, which aids clinicians in fast diagnosis. Artificial Neural Networks, Support Vector Machines, Radial Basis Function Neural Networks, Decision Trees, Computational Neural Networks, and various optimisation techniques have been used in different studies. The progression of keratoconus is identified by analyzing the shape of the cornea with Local Binary Pattern (LBP), Local Directional Pattern (LDP), Local Optimal Oriented Pattern (LOOP), and Cat Swarm Optimization (CSO) to detect the changes in cornea edges. The image processing with the CSO algorithm optimizes the result for the changes in the cornea and keratoconus detection. A new automated solution for detecting keratoconus is presented that employs texture analysis techniques such as LBP, LDP, LOOP, and CSO. The CSO extracts morphological and granular features from images of the cornea. The proposed method can be used to detect keratoconus by identifying the cornea shape change and improving clinical decisions. Further research can be in the way of grading the level of keratoconus.
\end{abstract}

Keywords: Keratoconus, Local Binary Pattern, Local Directional Pattern, Local Optimal Oriented Pattern, Cat Swarm Optimization

\section{Introduction}

Keratoconus is an eye disease that causes gradual thinning of the cornea and variations in the cornea's shape. The cornea is the eye's clear and thin outer layer and has a dome shape. If the thinning of the cornea proceeds gradually, a cone-shaped bulge forms in the area of the greatest thinning. People with this disorder have distorted vision, sensitivity to light (photophobia), and additional vision problems. Keratoconus often occurs at puberty, and most often, young adults or teenagers have this disorder. The particular cause of this disease is not completely understood, and mostly the cause of the disorder is from the interaction of multiple factors, including environmental and genetic factors. One factor that can contribute to the cause of Keratoconus is eye rubbing. For treatment of Keratoconus, glasses or contact lenses should be provided early, while a few cases may require corrective surgery.

The corneas in both eyes are generally infected (bilateral), although the development and severity of the condition in every eye may vary (asymmetric development). Symptoms generally become apparent during adolescence or young adulthood. Keratoconus may become increasingly worse for 10 to 20 years before slowing. Older adults particularly do not have worsening of keratoconus. Because of this eye disorder's progressive nature, infected individuals should change glasses frequently. It is important to diagnose the infected cornea to provide appropriate treatment. Therefore, efforts should be made to enhance the software and hardware to present devices that yield precise readings. There are various ways to detect keratoconus. The most used one is Pentacam, a device that provides cornea maps and 
information about the cornea's health. The four refractive maps such as Sagittal, Pachymetric, Elevation front, and Elevation back maps help detect keratoconus, and each map indicates the eye. The analysis of 4 maps aids in keratoconus diagnosis. In this paper, keratoconus is diagnosed by determining the cornea's shape through a computer-aided algorithm to delineate the cornea region.

The keratoconus is a corneal tissue degeneration caused by a progressive asymmetry, increasing corneal curvature and thinning. The keratoconus index (KI) is a new biometric parameter used in the diagnosis of Keratoconus. A semi-automatic technique for enhancing the KI computation uses images obtained from an ultrasound bio-microscope [1]. Corneal topography is utilized to measure the cornea's anterior surface. The cornea's anterior surface is generally represented as radial slope, radial curvature, and elevation. The mean curvature mapping application provides corneal topographies. The keratoconus and other diseases are characterized by a local increase in corneal curvature [2]. Keratoconus is particularly related to the decrease in visual acuity. Due to the wide prevalence of keratoconus, the requirement is not satisfied for developing new tools that can diagnose the disease at an early stage to prevent disease progression and vision loss. This research aims to develop and test a machine-learning algorithm that can detect keratoconus at early stages [3].

Keratoconus is difficult to identify in the early stages, as the patient does not feel any pain. Hence, the development of a Keratoconus Detection (KD) technique using a digital image processing technique is required for the early detection of Keratoconus so that physicians can provide patients with the required treatment in the earlier stage itself. The purpose of this research is to develop a Keratoconus Detection Technique utilizing a camera from a smart device to obtain anterior and lateral segment photographed eye images (A\&LSPIs) [4]. Corneal cross-linking (CXL) uses image analysis and machine learning to automatically identify and measure cornea's demarcation line presence and depth in OCT images. The automated technique provides the user with haze statistics and visual annotation, reflecting the haze's shape, location, and demarcation line in the cornea [5].

The computation of the true mean curvature over every point of the central region of the cornea is presented. The true mean curvature can precisely identify the keratoconus's location. A quartic smoothing spline algorithm computes the elevation maps for anterior and posterior corneal surfaces, true mean curvature, and pachymetry. The input to the algorithm is data from a single measurement from imaging devices such as a scheimpflug imager or an anterior segment optical coherence tomographer [6]. Tissue's mechanical properties can provide useful information on the integrity and health of tissues and help diagnose and track the progression of keratoconus. Optical coherence elastography (OCE) is a rapidly evolving technique that analyzes localized mechanical contrast in spatial resolution micrometer tissues. Noncontact optical coherence elastography measures the variations in the cornea's mechanical properties after UV-induced collagen cross-linking [7].

The cornea's noninvasive measurement of in-vivo mechanical properties is envisaged to find many applications in ophthalmology. Such high-resolution local corneal stiffness measurements may lead to better predicting and interpreting corneal pathologies such as keratoconus. These high-resolution measurements may also provide a quantitative assessment of corneal biomechanical response after corneal refractive surgery and a technique for evaluating the effectiveness of advanced corneal therapies such as treatment based on Riboflavin/UltraViolet-A Connecting Corneal Cross (UVA CXL) or femtosecond laser corneal transplantation. Here the corneal elasticity is evaluated using high-resolution imaging such as supersonic shear imaging [8]. The most common approach to measuring corneal topography is the Placido disk system, based on projecting an illuminated pattern of concentric rings or mires onto the cornea's surface. The concentric rings' image is captured and digitized with a fixed number of meridians, providing several thousands of points close to the concentric pattern. A simple Bayesian network classifier is presented to evaluate normal and keratoconus eyes samples for keratoconus identification. This utilizes previously developed topographic indices computed directly from the digital analysis of the Placido ring images [9].

The corneal thickness is one of the significant corneal parameters in diagnosing corneal ectasia especially early grades of keratoconus. One of the most specific characteristics of keratoconus is noninflammatory corneal thinning. In this disorder, the corneal thickness measurement is essential for diagnosing, staging, monitoring, and proper planning for surgical treatment. Ultrasound pachymetry is a well-known technique for measuring central corneal thickness (CCT), but it is invasive, and errors occur due to incorrect placement of the probe and fixation errors. Therefore, other devices based on technologies such as Specular microscopy, Optical Coherence Tomography (OCT), Ultrasound Biomicroscopy, Slit Scanning Topography (Orbscan), Scheimpflug Imaging (Pentacam), and Dual-Beam Partial Coherence Interferometry are utilized in clinical applications to find the repeatability of corneal 
thickness measurements with and Slit Scanning (Orbscan), Scheimpflug (Pentacam) imaging techniques in different grades of keratoconus [10].

Corneal topography is the main diagnostic technique for detecting and pachymetry data, and corneal aberrations are also generally used. Recently, tomographic evaluations using Optical Coherence Tomography (OCT) and corneal biomechanical indices have been utilized. In incipient and subclinical keratoconus, a single parameter used as a diagnostic factor is not precise enough. In these situations, the application of predictive models and algorithms is essential. Additionally, finding whether the disease will progress is significant for selecting the most suitable treatment. Certain factors, such as age, keratometric indices, corneal thickness, and corneal elevation data, can be useful to predict the keratoconus progression [11]. Advanced keratoconus clinical diagnosis is not complex because the classic bio-microscopic and retinoscopic signs are present in the advanced stages. Since the introduction of corneal topography, many techniques have been presented to differentiate between normal and keratoconus eyes. Color-coded maps' qualitative analysis allows advanced keratoconus's direct pattern recognition but is biased by subjective interpretation in earlier cases. Hence, a new classification technique is needed to diagnose keratoconus based on corneal measurements obtained from a Scheimpflug camera combined with Placido Corneal Topography [12].

Activating latent biomechanical instability in the subclinical keratoconus is the primary cause of corneal ectasia after refractive surgery. Biomechanical corneal destabilization in keratoconus eyes may be present and may be identifiable before the disease's tomographic and clinical signs until the manifestation of topographical changes. Corneal biomechanical properties may have the ability to overcome the existing gap in early or marginal types of keratoconus detection. Here, the aim is to test the diagnostic capacity of Corneal Visualization Scheimpflug Technology (Corvis ST) using corneal biomechanical parameters in keratoconus corneas [13]. Viscoelastic corneal properties depend on the cornea's internal structure and composition. They vary among subjects with respect to several factors, such as gender, age, ethnicity, certain hormones presence, and illnesses directly related to collagen synthesis. The main objective of the current research is to identify the essential parameters provided by non-contact tonometry for the cornea's biomechanical characterization and find from them an optimized function based on modified variables in IOP and CCT. This is done to differentiate between subclinical keratoconus eyes and healthy eyes [14].

Detecting moderate and advanced keratoconus is not complex using Corneal Topography, Biomicroscopic, Retinoscopic and Pachymetric findings. Many indices have been presented to aid in diagnosing keratoconus and subclinical keratoconus with various topography systems. They include quantitative descriptors such as the Keratometry (K), Inferior or Superior (I-S) value, Astigmatism (KISA) \% index. For assessing the anterior segment parameters of keratoconus (KC) eyes at various stages of the disease, a new Scheimpflug camera combined with Placido disk corneal topography is used [15].

\section{Materials and methods}

The function of the cornea serves as the eye's most powerful lens that bends incoming light onto the lower-powered internal lens, where the light is then passed to the retina. The retina converts light to signals. The signals are carried by the optic nerve to the brain to form images. The cornea should remain clear and in proper shape to transmit and focus the incoming light. In the case of Keratoconus, the abnormal cone shape causes the cornea to focus light inappropriately on the retina. Keratoconus may initially cause slight blurring of vision, increased sensitivity to bright light, and cause visual difficulty at low illumination. Some individuals may have double vision, partial vision, and an incomplete image around what they look. In certain cases, there may be a loss of vision clarity. With gradual corneal changes, affected individuals may have more difficulty seeing farther objects and have decreased vision clarity. Overall, vision changes can vary from 1 person to another. The severity can range from mild vision loss to more severe vision loss that causes a reduced ability to see even with corrective lenses. Although the diagnosis and grading of keratoconus have been advanced using various machine learning techniques, the classification between keratoconus and forme fruste keratoconus has been less accurate and needs to be enhanced. The feature vectors are extracted and given as input to support vector machines and other techniques for enhanced accuracy of classification of forme fruste keratoconus. The feature vector extraction is done using Local Binary Pattern (LBP), Local Directional Pattern (LDP) Local Optimal Oriented Pattern (LOOP) and CAT swarm optimization (CSO). 


\section{Local Binary Pattern (LBP)}

The Local Binary Pattern (LBP) proposed by Ojala et al. [22] is a simple and efficient texture operator which labels the pixels of an image by thresholding the neighborhood of each pixel and considers the result as a binary number. The LBP represented by Eq. (1);

$\operatorname{LBP}_{P, R}=\sum_{p=0}^{P-1} s\left(g_{p}-g_{c}\right) 2^{p}, s(x)= \begin{cases}1, & x=0 \\ 0, & x<0\end{cases}$

where, $g_{c}$ - represents intensity of the grey value of the center pixel.

$g_{p}$ - represents intensity of neighboring pixel with index $p$-values.

$P$ - number of sampling points on a circle of radius $R$ (circular neighborhood).

$R$ - determines the spatial resolution of the method.

For a given pixel ' $g_{c}$ '. The gray values of neighbor pixels which do not fall exactly in the center of a pixel are estimated by interpolation. The texture image assessed by finding LBP pattern of each pixel in image represented by Eqs. (2) - (3);

$H(k)=\sum_{\mid i=1}^{I I} \sum_{\mid j=1}^{\mid J}|f|\left(L B P_{P, R}(i, j), k\right), k \in[0, K]$

$f(x, y)=\left\{\begin{array}{c}1, x=y \\ 0, \text { otherwise }\end{array}\right.$

where, $\mathrm{K}$ signifies the value of LBP pattern.

The value of ' $U$ ' in LBP value signifies the number of spatial binary pixel value in image. The change in binary pixel values represent by Eq. (4);

$$
\begin{array}{cc}
U\left(\mathrm{LBP}_{P, R}\right)= & \left|s\left(g_{P-1}-g_{c}\right)-s\left(g_{0}-g_{c}\right)\right| \\
& +\sum_{p=1}^{P-1}\left|s\left(g_{p}-g_{c}\right)-s\left(g_{p-1}-g_{c}\right)\right|
\end{array}
$$

The uniform LBP pattern will give minimal discontinuities in circular binary image. The LBP with uniform pattern gives different output with $2^{\mathrm{P}}$ probabilities. The rotation invariance pattern is denoted by Eq. (5);

$\operatorname{LBP}_{P, R}^{\text {riu } 2}=\left\{\begin{array}{cc}\sum_{p=0}^{P-1} s\left(g_{p}-g_{c}\right), & \text { if } U\left(L B P_{P, R}\right) \leq 2 \\ P+1, & \text { otherwise }\end{array}\right.$

The variance in grey value between central and neighboring pixel help in image element description. The central pixel gray value further decomposes to illustrate sign and magnitude component of pixels in image.

\section{Local Directional Pattern (LDP)}

The Local Directional Pattern (LDP) proposed by Jabid et al. [23] is a descriptor that assigns 8-bit binary code for each pixel in the image. The 8-bit binary encoded image is divided with $3 \times 3$ region in an image with kirsch mask convolution in 8 directions.

The $3 \times 3$ region in an image gives 8 responses with $\mathrm{m}_{0}, \mathrm{~m}_{1}, \mathrm{~m}_{2}, \ldots, \mathrm{m}_{7}$. From the responses the top response is assigned as 1 and rest assigned ' 0 ' as signified by Eq. (6).

$C[f(x, y)]:=\left(c_{i}=1\right)$ if $0 \leq i \leq 7$ and $m_{i} \geq \Psi$

where, $\Psi=k^{\text {th }}(M)$ of eye.

The same procedure can be applied throughout the image to produce kirsch mask for cornea region

\section{Local Optimal Oriented Pattern (LOOP)}

The LBP and LDP have drawbacks such as the arbitrary sequence of binarization weights and empirical assignment of value to the threshold variable. The Local Optimal Oriented Pattern (LOOP) proposed by Chakroborti et al. [24] overcomes these drawbacks by preserving the strength using 
nonlinear amalgamation of LBP and LDP. The kirsch mask in LDP provides the pixel intensity difference towards neighborhood pixels of $3 \times 3$ region. As used in LDP, the 8 kirsch mask uses pixels extending from $(\mathrm{n}=0,1, \ldots, 7)$ to determine the strength of intensity variation.

A binarization weight is assigned to each neighborhood pixels corresponding to kirsch output towards each pixel direction. The binarization weight indicates the possibility of occurrence of an edge in a specific direction. The 8 responses of kirsch mask with equivalent pixels intensity are assigned with exponential weights $\left(\mathrm{w}_{\mathrm{n}}\right)$ and rank of magnitude $\left(\mathrm{m}_{\mathrm{n}}\right)$ for cornea region.

The cornea region pixel LOOP value is represented by;

$\operatorname{LOOP}\left(x_{c}, y_{c}\right)=\sum_{n=0}^{7} s\left(i_{n}-i_{c}\right) \cdot 2^{w_{n}}$

where $\left(x_{c}, y_{c}\right)$ represents pixel intensity of corneal region in the image.

$i_{c}$ represents the intensity of image.

And;

$s(x)=\left\{\begin{array}{cc}1 & \text { if } x \geq 0 \\ 0 & \text { otherwise }\end{array}\right.$

\section{Cat Swarm Optimization (CSO)}

For a given problem, there might be any number of solutions available in the defined search space. Searching and checking the entire search space is computationally resource intensive. Nature-inspired algorithms are being used to improve the search. Trajectory-based and population-based algorithms are being developed. In trajectory-based algorithms, only one agent is employed to search for the optimal solution, whereas in population-based algorithms multiple agents are used to search for the solution [25]. Cat Swarm Optimisation (CSO) is one such population-based algorithm proposed by Chu et al. [26]. Many variants of CSO like Discrete Binary Cat Swarm Optimisation (BCSO), Multiobjective Cat Swarm Optimisation (MOCSO), Parallel Cat Swarm Optimisation (PCSO), Enhanced Parallel Cat Swarm Optimisation (EPCSO), Average Inertia Weighted Cat Swarm Optimisation (AICSO), and Adaptive Dynamic Cat Swarm Optimisation (ADCSO) have been implemented in the literature. Cat Swarm Optimisation has also been implemented in combination with various Neural Networks. Here we have considered the basic Cat Swarm Optimisation proposed by Chu et al. [26] for extracting the features of the cornea.

The CSO algorithm is based on the behavior of cats. The cats appear to remain still in a place, are in a state of alertness, observing the surroundings, and once they see a target, they move rather quickly. Multiple cats are deployed in the algorithm wherein each cat has;

a) a position of its own with $\mathrm{M}$ dimensions and corresponding velocities,

b) a fitness value used to represent the goodness of the solution and

c) a flag for identifying the mode as tracing or seeking mode.

The number of cats to be engaged in an iteration is specified, where the algorithm is run. For each iteration, the best cat is stored, and the cat at the end of the final iteration is used to represent the final solution. The algorithm consists of the following steps;

a) The bounds, both upper and lower, are specified for the solution set

b) $\mathrm{N}$ cats or solution sets are randomly generated and are spread in the search space

c) Based on the Mixture Ration (MR), the cats are classified as into either tracing mode or seeking mode

d) The fitness values are measured, and best cat chosen to be saved in the memory

e) The cats are then redistributed in the next iteration depending on the mixture ratio.

The seeking mode represents the resting state of cats. Four parameters are used in this mode namely;

a) Seeking Memory Pool (SMP)

b) Seeking Range of the selected Dimension (SRD)

c) Counts of Dimensions to Change (CDC) and

d) Self Position Considering (SPC) [26].

Step 1: The current position of cat is $\mathrm{j}$ copies where $\mathrm{j}=\mathrm{SMP}$. If the value of SPC is set to true, then the value of $j=(S M P-1)$ then it retains the current position as one of the candidate. 
Step 2: For each copy, the SRD values either positively or negatively replace random values with respect to CDC.

Step 3: Evaluate the fitness value obtained for all candidates' position.

Step 4: If fitness value is not equal then set all the selecting probability of each candidate points with respect to Eq. (1). If fitness values are equal then each candidate is assigned with probability 1 represented by Eq. (9).

$P_{i}=\frac{\left|F S_{i}-F S_{b}\right|}{F S_{\max }-F S_{\min }}$

The present cat probability (pi) is stated by the ratio of difference between the minimum and maximum fitness function $\left(\mathrm{FS}_{\max }\right)$ and $\left(\mathrm{FS}_{\min }\right)$ for cat $\mathrm{I}$ fitness value $\left(\mathrm{FS}_{\mathrm{i}}\right), \mathrm{FS}_{\max }$ for minimization and maximization $\left(\mathrm{Fs}_{\mathrm{b}}\right)$.

Step 5: the current cat position is replaced by randomly selecting a new candidate point to move.

The goal is to find the minimum solution of fitness function given by $\mathrm{FS}_{\mathrm{b}}=\mathrm{FS}_{\max }$, otherwise, the maximum solution of fitness function is $\mathrm{FS}_{\mathrm{b}}=\mathrm{FS}_{\text {min }}$.

The tracking state mode is used for tracing the targets of granular region in cornea. But conventional wavelet changes do not extricate granular features. In tracking mode, the cat moves according to its own velocity from 1 dimension to the other.

Step 1: Initially update the velocity of every dimension with respect to (vk,d)

$v_{k, d}=v_{k, d}+r_{1} \times c_{1} \times\left(x_{b e s t, d}-x_{k, d}\right)$, where $d=1,2, \ldots, M$

where, $x_{b e s t, d}$ denotes position of cat with best fitness value,

$x_{k, d}$ denotes the position of cat,

$c_{l}$ is constant,

$r_{l}$ is a random variable range between $[0,1]$.

Step 2: If the velocities are above maximum velocity. And if the new velocity is over-range then the particular velocity is set to be equal.

Step 3: The position of cat (catk) updated with regard to Eq. (11);

$x_{k, d}=x_{k, d}+v_{k, d}$

\section{Results and discussion}

The corneal images are processed with Local Binary Pattern (LBP), Local Directional Pattern (LDP), and Local optimal oriented pattern (LOOP). The texture analysis shows a change in pixel intensity values for changes in the cornea. The algorithm varies binary weights across the cornea and delineates the cornea. However, the corneal changes in the eyes affected with keratoconus are not visible due to minimal change in pixel variations, as shown in Figures 1 - 3. The keratoconus affected cornea changes in pixel intensity values for progressive thinning of the cornea and changes in cornea's shape. Hence, the irregular shaped of the cornea delineates with CSO. The CSO outlines and coordinates with respect to variation in cornea's shape. The CSO delineates the edges of the irregular cornea by analysing the texture. In grayscale image, the CSO algorithm in seeking mode looks for $\mathrm{a}^{*}(\mathrm{x})$ pixel value in the edges of the cornea. The $\mathrm{a}^{*}(\mathrm{x})$ pixel is different from an average pixel value of surrounding pixel $\mathrm{b}^{*}(\mathrm{y})$ and Gaussian noise. In tracing mode, the CSO moves to neighbourhood pixels depending on pixel intensity and features of the cornea shape. However, due to minimal change in average pixel intensity value in cornea edges, the delineation becomes easier in grayscale image. The CSO in seeking mode, chooses the pixels with maximum intensity for delineation. The magnitude of granules have ' $n$ ' dimensional order vectors compared to cornea. The magnitude of ' $n$ ' dimension order vectors pixel value rank in ascending order. The pixel values threshold to select pixels associated with changes in the intensity of the pixels in the cornea edges. The CSO in tracing mode, (Figure 4) tracks down pixel with desired velocity for cornea edges and granule delineation. The feature vectors are to be used as inputs for training machine learning algorithms for classification and grading. Figure 5 compares the outputs of the LBP, LDP, LOOP and CSO outputs. The feature vectors are to be used for training classifiers. Techniques like Support Vector Machines, Neural Networks, and Convolutional Neural Networks are to be used and performances analysed as further research. 


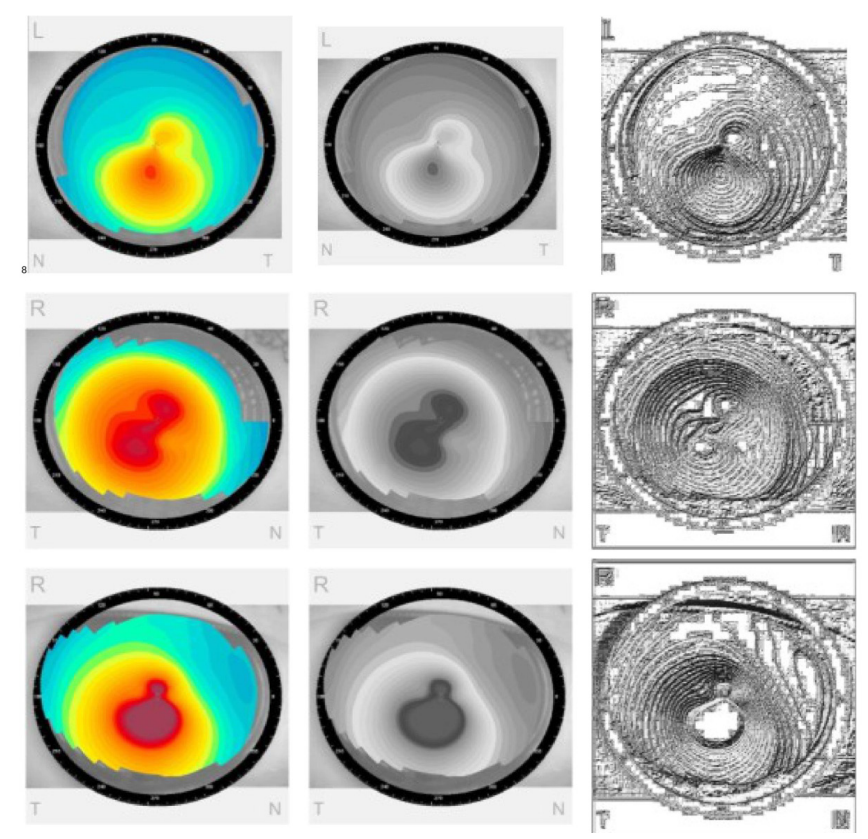

Figure 1 Local Binary Pattern (Columnwise): Input, grayscale and output images.

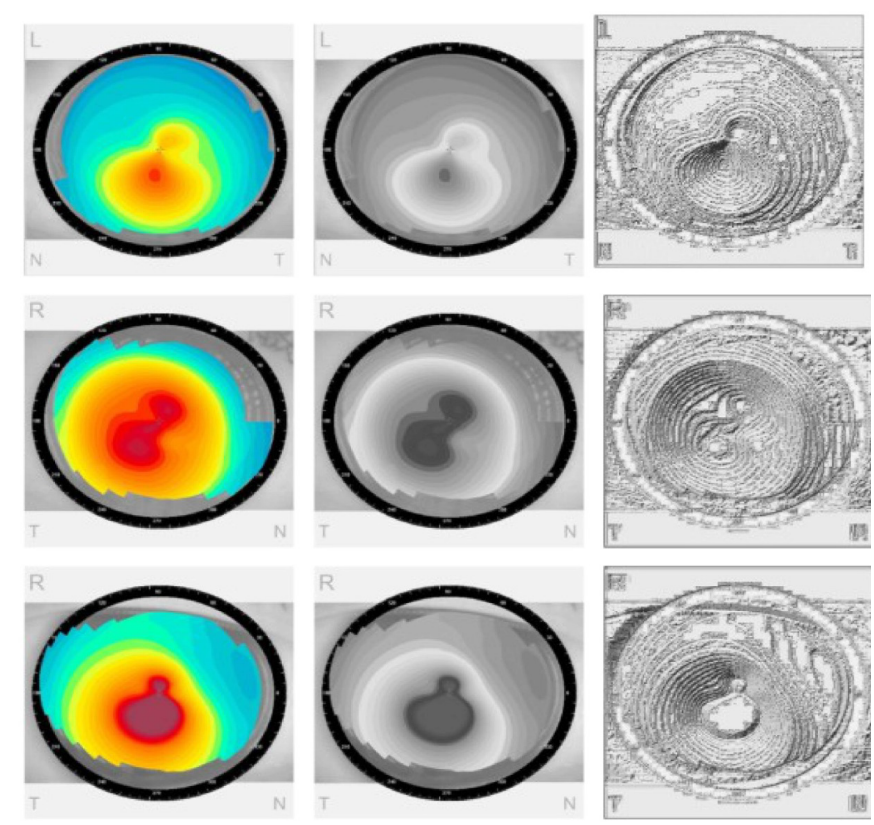

Figure 2 Local Directional Pattern (Columnwise): Input, grayscale and LDP Images. 

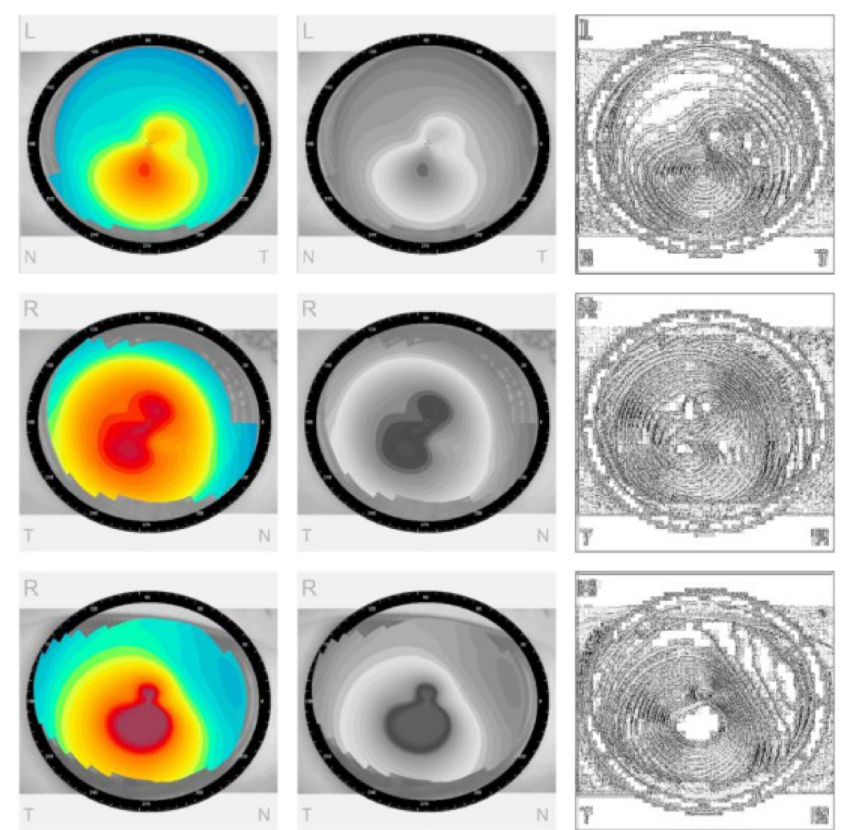

Figure 3 Local Optimal Oriented Pattern (Columnwise): Input, grayscale and LOOP images.

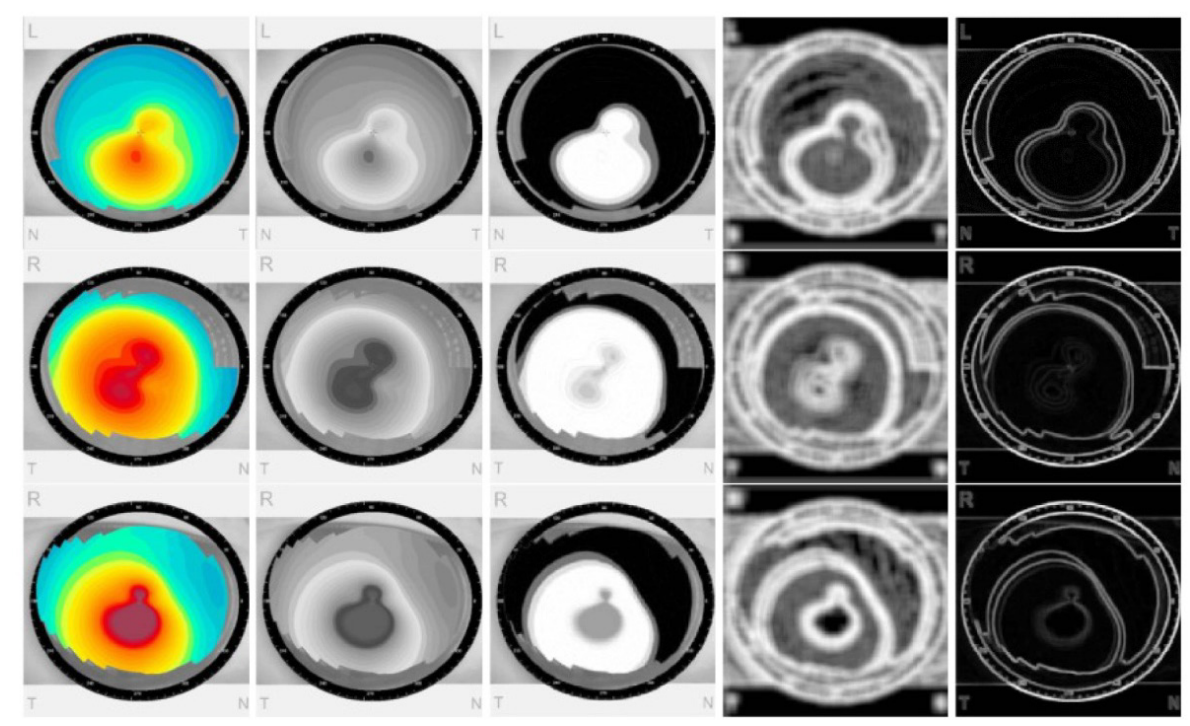

Figure 4 Cat Swarm Optimisation (Columnwise): Input, grayscale, seeking mode, tracking mode and CSO images. 


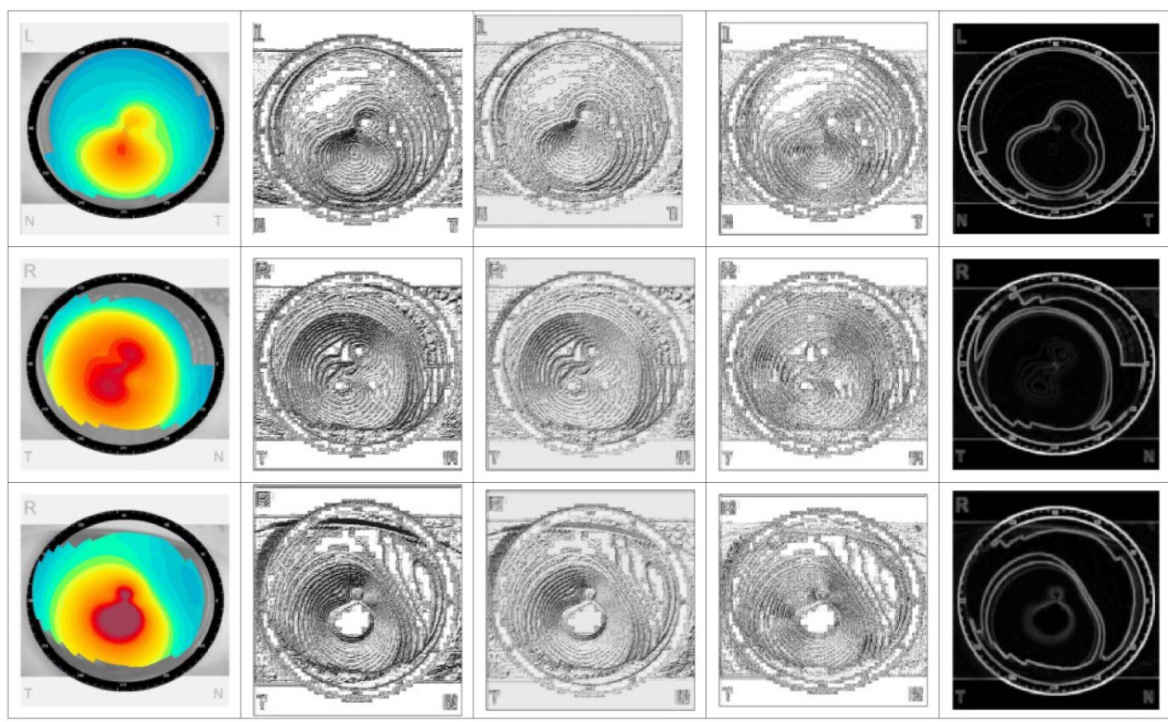

Figure 5 Comparison of schemes (Columnwise): Input, LBP, LDP, LOOP and CSO images

Diagnosis of Keratoconus and its grading has seen more advancements with the advent of artificial neural networks, machine learning, and optimisation algorithms. Different network architectures and algorithms, including Neural Networks, Radial Basis Forward Neural Networks, Multilayer Perceptron, Convolutional networks, Recurrent Neural Networks, and Support Vector Machines have been employed in the literature.

Accordo has used a three layered neural network with back propagation. et al. [16] for classification as normal, keratoconus, and others. With varying network sizes, learning rate parameters, and input parameters, global sensitivity of 81.7 to $94.1 \%$ and global accuracy of 92.9 to $96.1 \%$ have been achieved. The network performed better when provided with parameters from both eyes of the same patient than single eye parameters. Also, the network is not able to classify the forme fruste or subclinical keratoconus accurately.

Souza et al. [17] have used selective indices obtained from Orbscan II, of a single eye to classify as normal, astigmatism, keratoconus, and post-refractive surgery. The techniques employed are Radial Basis Function Neural Network (RBFNN), Support Vector Machine (SVM), and Multilayer Perceptron (MLP). The three machine learning techniques achieved good accuracy in distinguishing keratoconus and normal eyes.

Multi-Layer Perceptron (MLP), RBFNN, Neural Network (NN), Support Vector Machine (SVM), and Decision Tree (DT) have been employed by Toutounchian et al. [18] with two sets of features, the first set of 9 features obtained directly from Pentacam and the second set of 3 features obtained from the analysis of topographical images obtained from Pentacam.

The classification as abnormal, keratoconus, subclinical keratoconus, and normal eyes has been implemented by Arbelaz et al. [19] using a support vector machine. The inputs on having clinicians have diagnosed four groups of eyes: clinically diagnosed keratoconus, clinically diagnosed sub keratoconus, eyes undergone refractive surgery, and normal eyes. The precision of classification increases in the distinction between normal and subclinical cases when posterior corneal surface data are included along with anterior corneal surface data, while the accuracy decreases with the classification of sub-clinical keratoconus.

Correlation-based hierarchical clustering has been employed by Hidalgo et al. [20] to the Pentacam parameters, leading to a dendrogram followed by the selection of 1 variable from each branch, resulting in 22 parameters. The parameters thus obtained are then applied to a support vector machine for classification as normal, astigmatism, post-refractive surgery, forme fruste, and keratoconus. With the usage of topography maps from both anterior and posterior corneal surfaces, the performance in terms of sensitivity is higher in the identification of keratoconus eyes and lower for forme fruste keratoconus eyes.

Lavric et al. [21] used topographic maps with color scales with steep curved areas represented in warm colors and flat areas represented by cold colors. A convolutional neural network has been used to extract and learn the features of a keratoconus eye. Dataset obtained from KTC synteye has been used, and an accuracy of $99.33 \%$ was obtained. 


\section{Conclusions}

Texture analysis techniques such as LBP, LDP, LOOP, and CSO have been employed to extract the feature vectors for identifying and classifying keratoconus. The CSO extracts morphological and granular features from images of the cornea. The average pixel intensity thresholding increases the accuracy of granular features extraction and classification. The extracted granular features provide new morphological features which aid in detecting the change in cornea shape. Our experimental results highlighted the effectiveness and efficiency of the auto solution, which enables ophthalmologists to obtain fast, accurate, and objective information on a change in cornea shape to detect keratoconus. The proposed solution can be employed as a standardized method for detecting keratoconus through identifying the change in cornea shape and may enhance clinical decisions. Different variations of the Cat Swarm Optimisation can be considered and performance compared. Further classification with high accuracy can be done with anterior and posterior corneal features of both eyes by using machine learning techniques, including Support Vector machines and Convolutional Neural Networks.

\section{References}

[1] F Castiglione. Estimating the keratoconus index from ultrasound images of the human cornea. IEEE Trans. Med. Imag. 2000; 19, 1268-72.

[2] M Tang, R Shekhar and D Huang. Mean curvature mapping for detection of corneal shape abnormality. IEEE Trans. Med. Imag. 2005; 24, 424-8.

[3] A Lavric, V Popa, H Takahashi and S Yousefi. Detecting keratoconus from corneal imaging data using machine learning. IEEE Access 2020; 8, 149113-21.

[4] MM Daud, WMDW Zaki, A Hussain and HA Mutalib. Keratoconus detection using the fusion features of anterior and lateral segment photographed images. IEEE Access 2020; 8, 142282-94.

[5] AR Dhaini, M Chokr, SM El-Oud, MA Fattah and S Awwad. Automated detection and measurement of corneal haze and demarcation line in spectral-domain optical coherence tomography images. IEEE Access 2018; 6, 3977-91.

[6] F Nasrin, RV Iyer and SM Mathews. Simultaneous estimation of corneal topography, pachymetry, and curvature. IEEE Trans. Med. Imag. 2018; 37, 2463-73.

[7] M Singh, J Li, S Vantipalli, S Wang, Z Han, A Nair, SR Aglyamov, MD Twa and KV Larin. Noncontact elastic wave imaging optical coherence elastography for evaluating changes in corneal elasticity due to crosslinking. IEEE J. Sel. Top. Quant. 2016; 22, 6801911.

[8] M Tanter, D Touboul, JL Gennisson, J Bercoff and M Fink. High-resolution quantitative imaging of cornea elasticity using supersonic shear imaging. IEEE Trans. Med. Imag. 2009; 28, 1881-93.

[9] GM Castro-Luna, A Martínez-Finkelshtein and D Ramos-López. Robust keratoconus detection with Bayesian network classifier for Placido-based corneal indices. Contact Lens Anterio Eye 2020; 43, 366-72.

[10] MA Amiri, H Hashemi, S Ramin, A Yekta, A Taheri, P Nabovati and M Khabazkhoob. Corneal thickness measurements with Scheimpflug and slit scanning imaging techniques in keratoconus. $J$. Curr. Opthalmol. 2017; 29, 23-7.

[11] A Martínez-Abad and DP Piñero. New perspectives on the detection and progression of keratoconus. J. Cataract Refract. Surg. 2017; 43, 1213-27.

[12] MC Arbelaez, F Versaci, G Vestri, P Barboni and G Savini. Use of a support vector machine for keratoconus and subclinical keratoconus detection by topographic and tomographic data. Ophthalmology 2012; 119, 2231-8.

[13] R Elham, E Jafarzadehpur, H Hashemi, K Amanzadeh, F Shokrollahzadeh, A Yekta and M Khabazkhoob. Keratoconus diagnosis using Corvis ST measured biomechanical parameters. $J$. Curr. Ophthalmol. 2017; 29, 175-81.

[14] P Peña-García, C Peris-Martínez, A Abbouda and JM Ruiz-Moreno. Detection of subclinical keratoconus through non-contact tonometry and the use of discriminant biomechanical functions. $J$. Biomech. 2016; 49, 353-53.

[15] M Safarzadeh and N Nasiri. Anterior segment characteristics in normal and keratoconus eyes evaluated with a combined Scheimpflug/Placido corneal imaging device. J. Curr. Ophthalmol. 2016; 28, 106-11.

[16] PA Accardo and S Pensiero. Neural network-based system for early keratoconus detection from corneal topography. J. Biomed. Inform. 2002; 35, 151-9. 
[17] MB Souza, FW Medeiros, DB Souza, R Garcia and MR Alves. Evaluation of machine learning classifiers in keratoconus detection from orbscan II examinations. Clinics (Sao Paulo) 2010; 65, 1223-8.

[18] F Toutounchian, J Shanbehzadeh and M Khanlari. Detection of keratoconus and suspect keratoconus by machine vision. In: Proceedings of the International Multi Conference of Engineers and Computer Scientists, Hong Kong, China. 2012.

[19] MC Arbelaez, F Versaci, G Vestri, P Barboni and G Savini. Use of a support vector machine for keratoconus and subclinical keratoconus detection by topographic and tomographic data. Ophthalmology 2012; 119, 2231-8.

[20] IR Hidalgo, P Rodriguez, JJ Rozema, SN Dhubhghaill, N Zakaria, MJ Tassignon and C Koppen. Evaluation of a machine-learning classifier for keratoconus detection based on scheimpflug tomography. Cornea 2016; 35, 827-32.

[21] A Lavric and P Valentin. KeratoDetect: Keratoconus detection algorithm using convolutional neural networks. Comput. Intell. Neurosci. 2019; 2019, 8162567.

[22] T Ojala, M Pietikainen and T Maenpaa. Multiresolution gray-scale and rotation invariant texture classification with Local Binary Patterns. IEEE Trans. Pattern. Anal. Mach. Intell. 2002; 24, 97187.

[23] T Jabid, MH Kabir and O Chae. Gender classification using Local Directional Pattern (LDP). In: Proceedings of the $20^{\text {th }}$ Internatioanl Conference on Pattern Recognition, Istanbul, Turkey. 2010.

[24] T Chakroborti, B McCane, S Mills and U Pal. LOOP descriptor: Local optimal-oriented pattern. IEEE Signal Process. Lett. 2018; 25, 635-9.

[25] AM Ahmed, TA Rashid and SAM Saeed. Cat swarm optimization algorithm: A survey and performance evaluation. Comput. Intell. Neurosci. 2020; 2020, 4854895.

[26] SC Chu, PW Tsai and JS Pan. Cat swarm optimisation. In: Proceedings of the Pacific Rim International Conference on Artificial Intelligence, Guilin, China. 2006, p. 854-8. 